\title{
The Dynamics of a Nonautonomous Predator-Prey Model with Infertility Control in the Prey
}

\author{
Xiaomei Feng, ${ }^{1,2}$ Zhidong Teng, $^{1}$ and Fengqin Zhang $^{2}$ \\ ${ }^{1}$ College of Mathematics and System Sciences, Xinjiang University, Urumqi 830046, China \\ ${ }^{2}$ Department of Mathematics, Yuncheng University, Yuncheng 044000, China
}

Correspondence should be addressed to Zhidong Teng; zhidong@xju.edu.cn

Received 16 December 2013; Accepted 20 January 2014; Published 10 March 2014

Academic Editor: Weiming Wang

Copyright (c) 2014 Xiaomei Feng et al. This is an open access article distributed under the Creative Commons Attribution License, which permits unrestricted use, distribution, and reproduction in any medium, provided the original work is properly cited.

\begin{abstract}
A nonautonomous predator-prey model with infertility control in the prey is formulated and investigated. Threshold conditions for the permanence and extinction of fertility prey and infertility prey are established. Some new threshold values of integral form are obtained. For the periodic cases, these threshold conditions act as sharp threshold values for the permanence and extinction of fertility prey and infertility prey. There are also mounting concerns that the quantity of biological sterile drug is obtained in the process of the prevention and control of pest in the grasslands and farmland. Finally, two examples are given to illustrate the main results of this paper. The numerical simulations shown that, when the pest population is permanet, different dynamic behaviors may be found in this model, such as the global attractivity and the chaotic attractor.
\end{abstract}

\section{Introduction}

Small mammals living in the grasslands, such as the plateau pika, not only burrow, but also accumulate the soil outside the hole, which makes the grass cease growing. More seriously, after a rainstorm, the soil would be washed away which increased soil erosion. So a greater range of damages resulted. And the lack of protective vegetation exacerbated the desertification and degradation of pastures. Besides, pirates of pikas also eat grass, which reduced the carrying capacity. When the number of these small mammals increased sharply, it would cause a lot of trouble and loss to economy, ecology, and people's lives on the grassland. So at this moment, they are referred to as harmful animals.

As the change of the natural environment by the human production activities, agricultural, and the rapid development of cities provide plenty of food resources and good habitat for rodent, rat increases seriously, the management of pest also will be more difficult. Mouse control strategy from the traditional damage caves and machinery catch to fumigation, acute rodenticide, anticoagulant therapy, and the application of many chemical methods has made important progress. At present, the chemical prevention and control play an important role in the mouse control technology. However, chemical control is effective for short and harmful rat will soon come again and reproduction rapidly leads to the quick rebound in this species. In the fields, the application of acute rodenticide reaches 80; the population in the two years can be restored to the original level. In addition, there still exist many problems such as environmental pollution, secondary poisoning, and fungicide resistance in chemical control, which makes chemical Rodenticide restricted to the application of the rodent sustainable control. And integrating multidisciplinary approach and means, the sterility control technology based on ecological security has gradually become the development direction of rodent control. Infertility control technology has both directly and indirectly reduced the rodent population density, and will not lead to sharp fluctuations in ecological system, so it has a very good advantage in the environmental safety and cost-effectiveness.

Now, there are very serious rat in many areas of China, such as Xinjiang, Inner Mongolia, Gansu, Shanxi, and The Tibetan Plateau. In the Inner Mongolia grasslands, it is predicted that pest harm area is about 100 million mu and the serious disaster area is about 50 million mu [1]. Prairie mousehole per hectare is 300 at least and even 900 at most. 
In 2006, eleven silver foxes were introduced for the first time at Alxa League in Inner Mongolia grassland in order to control the prairie mice. Those foxes can catch large amounts of prairie gerbil, Meriones unguiculatus, and jerboa [2]. In addition, in 2011, Beowulf Biological antisterility rodenticide was used. The purpose is to test the effect of preventing grassland rat and whether or not achieves these requirements such as restraining the birth rates of harmful rat population, reducing the pest population density, slowing population growth benefiting Environmental Health and Safety [3].

At present, the research about infertility control is at most laboratory studies [4-9] and theoretical analysis even less. Based on the above understanding of the facts and mathematical biology background, the study about a class of predatorprey model with infertility control in the prey (harmful rat) is very meaningful. Moreover, the result indicated that species and quantity are different by vegetation and physiognomy, and change of density is more distinct along with changing season. Therefore, it is a very basilic problem to research this kind of nonautonomous population dynamic systems.

It is interesting to note that rodents living in the North generally have seasonal breeding, such as plateau pika nearby Qinghai lake breed from April to August, Brandt's voles breed from March to September, and Mongolian gerbil in Inner Mongolia breed from April to August. Obviously, this kind of periodic phenomenon, extensively exists in the real world. Therefore, the dynamical behavior of the $\omega$-periodic system is also worthy of being discussed.

Now, we only consider infertility control in the prey (harmful rat) $X(t)$ population. It is composed of two population classes: one is the class of fertility prey, denoted by $F(t)$, and the other is the class of infertility prey, denoted by $S(t)$. Therefore, at any time $t$, the total density of prey population is $X(t)=F(t)+S(t)$. Fertility rodents will become infertile after eating the sterilant. Therefore, $\mu(t)$ is assumed to the rate at which infertility prey contacts occur. In this paper, we study the following nonautonomous predator-prey model with infertility control in the prey:

$$
\begin{gathered}
\frac{d F(t)}{d t}=F(t)\left[b_{1}(t)-a_{11}(t)(F(t)+S(t))\right. \\
\left.-\mu(t)-a_{12}(t) Z(t)\right] \\
\frac{d S(t)}{d t}=\mu(t) F(t)-d_{2}(t) S(t)-a_{11}(t) \\
\times[F(t)+S(t)] S(t)-a_{12}(t) S(t) Z(t) \\
\frac{d Z(t)}{d t}=Z(t)\left[b_{2}(t)+a_{21}(t)(F(t)+S(t))-a_{22}(t) Z(t)\right]
\end{gathered}
$$

with initial conditions

$$
F(0)=F_{0}, \quad S(0)=S_{0}, \quad Z(0)=Z_{0},
$$

where

$$
\left(F_{0}, S_{0}, Z_{0}\right) \in R_{+}^{3}=\left\{(F, S, Z) \in R^{3}: F>0, S>0, Z>0\right\} .
$$

Here, $F(t)$ is the fertility prey population density, $S(t)$ is the sterility prey population density, $Z(t)$ is the predator population density, $b_{1}(t), a_{11}(t)$ are the intrinsic growth rate and density-dependent coefficient of the prey, respectively, $b_{2}(t)$, $a_{22}(t)$ are the intrinsic growth rate and density-dependent coefficient of the predator, respectively, $a_{12}(t)$ is the capturing rate of the predator, and $a_{21}(t)$ is the rate of conversion of nutrients into the reproduction of the predator.

\section{Preliminaries}

For a continuous bounded function $f(t)$ defined on $R_{+}=$ $[0, \infty)$, we denote

$$
f^{m}=\limsup _{t \rightarrow \infty} f(t), \quad f^{l}=\liminf _{t \rightarrow \infty} f(t) .
$$

If $f$ is $\omega$-periodic, then the average value of on a time interval $[0, \omega]$ can be defined as

$$
\bar{f}=\frac{1}{\omega} \int_{0}^{\omega} f(t) d t
$$

For system (1), we introduce the following assumptions.

$\left(\mathrm{H}_{1}\right)$ Functions $a_{11}(t), \mu(t), a_{12}(t), d_{2}(t), a_{21}(t)$, and $a_{22}(t)$ are all negative, continuous, and bounded on $R_{+}$and $b_{i}(t)(i=1,2)$ are continuous and bounded functions.

$\left(\mathrm{H}_{2}\right)$ There exist positive constants $\omega_{j}>0(j=1,2,3$, $4,5,6)$ such that

$$
\begin{array}{cc}
\liminf _{t \rightarrow \infty} \int_{t}^{t+\omega_{i}} b_{i}(\theta) d \theta>0, & \liminf _{t \rightarrow \infty} \int_{t}^{t+\omega_{3}} d_{2}(\theta) d \theta>0, \\
\liminf _{t \rightarrow \infty} \int_{t}^{t+\omega_{3+i}} a_{i i}(\theta) d \theta>0, & \liminf _{t \rightarrow \infty} \int_{t}^{t+\omega_{6}} \mu(\theta) d \theta>0 .
\end{array}
$$

In particular, when model (1) degenerates into $\omega$-periodic system, that is, $a_{11}(t), \mu(t), a_{12}(t), d_{2}(t), a_{21}(t), b_{1}(t), b_{2}(t)$, and $a_{22}(t)$ are continuous periodic functions with period $\omega>0$, then assumption $\left(\mathrm{H}_{2}\right)$ is equivalent to the following forms:

$$
\left(\overline{\mathrm{H}_{2}}\right) \overline{b_{i}}>0, \overline{a_{i i}}>0, \overline{d_{2}}>0 \text {, and } \bar{\mu}>0(i=1,2) \text {. }
$$

In the following, we state several lemmas which will be useful in the proof of main results in the paper.

Firstly, we consider the following nonautonomous logistic equation:

$$
\frac{d z(t)}{d t}=z(t)\left(b_{2}(t)-a_{22}(t) z(t)\right)
$$

where functions $b_{2}(t)$ and $a_{22}(t)$ are bounded continuous defined on $R_{+}$and $a_{22}(t) \geq 0$ for all $t \geq 0$. We have the following result.

Lemma 1 (see [10]). Suppose that there are constants $\omega_{i}>$ $0(i=1,2)$ such that

$$
\liminf _{t \rightarrow \infty} \int_{t}^{t+\omega_{1}} b_{2}(\theta) d \theta>0, \quad \liminf _{t \rightarrow \infty} \int_{t}^{t+\omega_{2}} a_{22}(\theta) d \theta>0 .
$$


Then,

(a) there exist positive constants $m$ and $M$ such that for any positive solution $z(t)$ of $(7)$

$$
m \leq \liminf _{t \rightarrow \infty} z(t) \leq \limsup _{t \rightarrow \infty} z(t) \leq M ;
$$

(b) each fixed positive solution $z^{*}(t)$ of (7) is globally uniformly attractive;

(c) if $a_{22}^{l}>0$, then for any positive solution $z(t)$ of (7)

$$
\left(\frac{b_{2}}{a_{22}}\right)^{l} \leq \liminf _{t \rightarrow \infty} z(t) \leq \limsup _{t \rightarrow \infty} z(t) \leq\left(\frac{b_{2}}{a_{22}}\right)^{m} ;
$$

(d) if (7) is $\omega$-periodic, then condition (8) reduces to $\overline{b_{2}}>0$ and $\overline{a_{22}}>0$; thus (7) has a uniformly attractive positive $\omega$-periodic solution. tion:

Further, we consider the following nonautonomous equa-

$$
\frac{d z(t)}{d t}=z(t)\left[b_{2}(t)-a_{22}(t) z(t)+a_{21}(t)(F(t)+S(t))\right],
$$

where $b_{2}(t)$ and $a_{22}(t)$ are defined as in (7) and $a_{21}(t)(F(t)+$ $S(t))$ is continuous and bounded function defined on $R_{+}$.

Let $z\left(t, t_{0}, z_{0}\right)$ be the solution of (11) with initial condition $z\left(t_{0}\right)=z_{0}$ and let $z_{0}(t)$ be some fixed positive solution of (7). We have the following result.

Lemma 2 (see [11]). Suppose that all conditions of Lemma 1 hold. Then for any constants $\varepsilon>0$ and $M>0$ there exist constant $\delta=\delta(\varepsilon)>0$ and $T=T(\varepsilon, M)>0$ such that for any $t_{0} \in R_{+}$and $z_{0} \in\left[M^{-1}, M\right]$, when $\left|a_{21}(t)(F(t)+S(t))\right|<\delta$ for all $t \geq t_{0}$, one has

$$
\left|z\left(t, t_{0}, z_{0}\right)-z_{0}(t)\right|<\varepsilon, \quad \forall t \geq t_{0}+T .
$$

Next, we consider the following nonautonomous linear equation:

$$
\frac{d u(t)}{d t}=\mu(t)-d_{2}(t) u(t),
$$

where $\mu(t)$ and $d_{2}(t)$ are bounded continuous defined on $R_{+}$ and $\mu(t) \geq 0$ for all $t \geq 0$. We have the following result.

Lemma 3 (see [12]). Suppose that there are constants $\omega_{i}>$ $0(i=1,2)$ such that

$$
\liminf _{t \rightarrow \infty} \int_{t}^{t+\omega_{1}} \mu(\theta) d \theta>0, \quad \liminf _{t \rightarrow \infty} \int_{t}^{t+\omega_{2}} d_{2}(\theta) d \theta>0 .
$$

Then,

(a) there exist positive constants $m$ and $M$ such that for any positive solution $u(t)$ of (13)

$$
m \leq \liminf _{t \rightarrow \infty} u(t) \leq \limsup _{t \rightarrow \infty} u(t) \leq M ;
$$

(b) each fixed positive solution $u^{*}(t)$ of (13) is globally uniformly attractive;

(c) if $d_{2}^{l}>0$, then for any positive solution $u(t)$ of (13)

$$
\left(\frac{\mu}{d_{2}}\right)^{l} \leq \liminf _{t \rightarrow \infty} z(t) \leq \limsup _{t \rightarrow \infty} z(t) \leq\left(\frac{\mu}{d_{2}}\right)^{m}
$$

(d) if (13) is $\omega$-periodic, then the condition (14) reduces to $\bar{\mu}>0$ and $\overline{d_{2}}>0$; thus (13) has a uniformly attractive positive $\omega$-periodic solution.

Further we investigate the following nonautonomous linear equation:

$$
\frac{d u(t)}{d t}=\mu(t)-d_{2}(t) u(t)+e(t),
$$

where $\mu(t)$ and $d_{2}(t)$ are defined as in (13) and $e(t)$ is continuous and bounded function defined on $R_{+}$.

Let $u\left(t, t_{0}, u_{0}\right)$ be the solution of (17) with initial condition $u\left(t_{0}\right)=u_{0}$ and let $u_{0}(t)$ be some fixed positive solution of (13). We have the following result.

Lemma 4 (see [13]). Suppose that there exists a constant $\gamma>0$ such that

$$
\liminf _{t \rightarrow \infty} \int_{t}^{t+\gamma} d_{2}(\theta) d \theta>0
$$

Then for any constants $\varepsilon>0$ and $M>0$ there exist constants $\delta=\delta(\varepsilon)>0$ and $T=T(\varepsilon, M)>0$ such that for any $t_{0} \in R_{+}$ and $u_{0} \in\left[M^{-1}, M\right]$, when $|e(t)|<\delta$ for all $t \geq t_{0}$, one has

$$
\left|u\left(t, t_{0}, u_{0}\right)-u_{0}(t)\right|<\varepsilon, \quad \forall t \geq t_{0}+T .
$$

In (17), if function $\mu(t) \equiv 0$, then we can obtain that $u_{0}(t) \equiv 0$. We have the following Corollary 5 of Lemma 4 .

Corollary 5. Suppose that $\mu(t) \equiv 0$ for all $t \in R^{+}$and there exists a constant $\gamma>0$ such that

$$
\liminf _{t \rightarrow \infty} \int_{t}^{t+\gamma} d_{2}(\theta) d \theta>0 .
$$

Then for any constants $\varepsilon>0$ and $M>0$ there exist constants $\delta=\delta(\varepsilon)>0$ and $T=T(\varepsilon, M)>0$ such that for any $t_{0} \in R_{+}$ and $u_{0} \in\left[M^{-1}, M\right]$, when $|e(t)|<\delta$ for all $t \geq t_{0}$, one has

$$
\left|u\left(t, t_{0}, z_{0}\right)\right|<\varepsilon, \quad \forall t \geq t_{0}+T .
$$

\section{Main Results}

It is obvious that the solution $(F(t), S(t), Z(t))$ of model (1) with initial condition (2) is positive; that is, $F(t)>0, S(t)>0$, $Z(t)>0$ for all $t \geq 0$ in the maximum interval of existence of the solution. On the ultimate boundedness of solutions of system (1), we get the following theorem. 
Theorem 6. Suppose that $\left(H_{1}\right)$ and $\left(H_{2}\right)$ hold. Then system (1) is ultimately bounded in the sense that there is a positive constant $M$ such that

$$
\begin{gathered}
\limsup _{t \rightarrow \infty} F(t)<M, \quad \limsup _{t \rightarrow \infty} S(t)<M, \\
\limsup _{t \rightarrow \infty} Z(t)<M
\end{gathered}
$$

for any positive solution $(F(t), S(t), Z(t))$ of system (1).

The ecological implication of Theorem 6 is that the fertility prey $F(t)$ is ultimately bounded. The sterility prey $S(t)$, when assumptions $\left(\mathrm{H}_{1}\right)$ and $\left(\mathrm{H}_{2}\right)$ hold, if $S(t)$ is not ultimately bounded, then $S(t)$ will expand unlimitedly. But the conversion of the fertile prey lies on the sterile prey by sterile drugs. So, the prerequisite for the unlimited increase of the sterility prey is that the fertility prey must be expanding unlimitedly. In short, the number of harmful rat will not go on rising forever.

Proof. Let $(F(t), S(t), Z(t))$ be any positive solution of system (1). From the first equation of system (1) we have

$$
\frac{d F(t)}{d t} \leq F(t)\left(b_{1}(t)-a_{11}(t) F(t)\right) .
$$

From $\left(\mathrm{H}_{2}\right)$, it is easy to verify that the comparison equation

$$
\frac{d x(t)}{d t}=x(t)\left[b_{1}(t)-a_{11}(t) x(t)\right]
$$

satisfies all conditions of Lemma 1. So, the comparison theorem and Lemma 1 imply that we obtain there is a constant $M_{1}$ such that for any positive solution $(F(t), S(t), Z(t))$ of system (1), there is a $T_{1}>0$ such that we have $F(t)<M_{1}$ for all $t \geq T_{1}$. Further, from the second equation of system (1) we have

$$
\frac{d S(t)}{d t} \leq \mu(t) M_{1}-d_{2}(t) S(t)
$$

for all $t \geq T_{2}$. From Lemma 3 it can be obtained that under assumption $\left(\mathrm{H}_{2}\right)$ any positive solution $x(t)$ of the following nonautonomous linear equation:

$$
\frac{d x(t)}{d t}=\mu(t) M_{1}-d_{2}(t) S(t)
$$

is ultimately bounded. Hence, using the comparison theorem, we further can obtain that there is a constant $M_{2}>0$ such that for any positive solution $(F(t), S(t), Z(t))$ of system (1), there is a $T_{2} \geq T_{1}$ such that $S(t)<M_{2}$ for all $t \geq T_{1}$. Lastly, from the third equation of equation of system (1) we have

$$
\frac{d Z(t)}{d t} \leq Z(t)\left(b_{2}(t)+a_{21}(t)\left(M_{1}+M_{2}\right)-a_{22}(t) Z(t)\right)
$$

for all $t \geq T_{2}$. Consider the following nonautonomous equation:

$$
\frac{d x(t)}{d t}=x(t)\left(b_{2}(t)+a_{21}(t)\left(M_{1}+M_{2}\right)-a_{22}(t) x(t)\right),
$$

the comparison theorem and Lemma 1 imply that there is a constant $M_{3}$ such that for any positive solution $(F(t), S(t), Z(t))$ of system (2), there is a $T_{3}>0$ such that $Z(t)<M_{3}$ for all $t \geq T_{3}$.

Now, let $M=\max \left\{M_{1}, M_{2}, M_{3}\right\}$; then from the above proofs, we have

$$
\begin{gathered}
\limsup _{t \rightarrow \infty} F(t)<M, \quad \limsup _{t \rightarrow \infty} S(t)<M, \\
\limsup _{t \rightarrow \infty} Z(t)<M .
\end{gathered}
$$

Therefore, solution $(F(t), S(t), Z(t))$ is ultimately bounded. This completes the proof.

Remark 7. Applying the comparison theorem and combining conclusion (c) of Lemmas 1 and 3, we can obtain that if assumptions $\left(\mathrm{H}_{1}\right)$ and $\left(\mathrm{H}_{2}\right)$ hold in system (1), $a_{11}^{l}>0, d_{2}^{l}>0$, and $a_{22}^{l}>0$, then constants $M_{i}(i=1,2,3)$ given above can be chosen by

$$
\begin{gathered}
M_{1}=\left(\frac{b_{1}}{a_{11}}\right)^{m}, \quad M_{2}=\left(\frac{\mu M_{1}}{d_{2}}\right)^{m}, \\
M_{3}=\left(\frac{b_{2}+a_{21}\left(M_{1}+M_{2}\right)}{a_{22}}\right)^{m} .
\end{gathered}
$$

Next, we discuss the permanence and extinction of fertility prey $F(t)$ and infertility prey $S(t)$.

Let $Z_{0}(t)$ be some fixed positive solution of the following nonautonomous logistic equation:

$$
\frac{d Z(t)}{d t}=Z(t)\left(b_{2}(t)-a_{22}(t) Z(t)\right)
$$

Particularly, if $a_{22}^{l}>0$, using conclusion (c) of Lemma 1, we can obtain

$$
\left(\frac{b_{2}}{a_{22}}\right)^{l} \leq \liminf _{t \rightarrow \infty} Z_{0}(t) \leq \limsup _{t \rightarrow \infty} Z_{0}(t) \leq\left(\frac{b_{2}}{a_{22}}\right)^{m} .
$$

Theorem 8. Suppose that $\left(H_{1}\right)$ and $\left(H_{2}\right)$ hold and there exists a constant $\lambda>0$ such that

$$
\liminf _{t \rightarrow \infty} \int_{t}^{t+\lambda}\left(b_{1}(\theta)-\mu(\theta)-a_{12}(\theta) Z_{0}((\theta))\right) d \theta>0 .
$$

Then,

$$
\liminf _{t \rightarrow \infty} F(t)>m, \quad \liminf _{t \rightarrow \infty} S(t)>m
$$

for any positive solution $(F(t), S(t), Z(t))$ of system (1). 
Theorem 8 shows that if we guarantee that assumptions $\left(\mathrm{H}_{1}\right),\left(\mathrm{H}_{2}\right)$ and $(35)$ hold, then the prey species must be permanent. In the ecological system, each component part, including the animal, plant and microorganism, plays its own role, and they are indispensable and irreplaceable. Every creature may deviate from its original trajectory, which lead to the outbreak of this population and the negative effect on human beings, such as harmful rat. Even if it happens, this species should not be extinct through the human activity. What we should do is to control the rat population to such a degree that will not be harmful to human beings. Therefore, the permanence of harmful rat given by Theorem 8 is very necessary.

Proof. Let $(F(t), S(t), Z(t))$ be any positive solution of system (1). From condition (17) there are positive constants $\varepsilon_{0}, \eta$ and $T^{*}$ such that for all $t \geq T^{*}$

$$
\begin{gathered}
\int_{t}^{t+\lambda}\left(b_{1}(\theta)-\mu(\theta)-2 \varepsilon_{0} a_{11}(\theta)-a_{12}(\theta)\right. \\
\left.\times\left(Z_{0}(\theta)+\varepsilon_{0}\right)\right) d \theta>\eta .
\end{gathered}
$$

According to Theorem 6 , there exists a constant $T^{* *} \geq T^{*}$ such that

$$
F(t)<M, \quad S(t)<M, \quad Z(t)<M,
$$

for all $t \geq T^{* *}$. Consider (11), that is,

$$
\frac{d z(t)}{d t}=z(t)\left(b_{2}(t)-a_{22}(t) z(t)+a_{21}(t)(F(t)+S(t))\right)
$$

from Lemma 2, for $\varepsilon_{0}$ and $M$ given in above there exist constants $\delta_{0}=\delta_{0}\left(\varepsilon_{0}\right)>0$ and $T_{0}=T_{0}\left(\varepsilon_{0}, M\right)>0$ such that for any $t_{0} \in R_{+}$and $Z_{0} \in[0, M]$, when $\left|a_{21}(t)(F(t)+S(t))\right|<$ $\delta_{0}$ for all $t \geq t_{0}$, we have

$$
\left|z\left(t, t_{0}, z_{0}\right)-z_{0}(t)\right|<\varepsilon_{0}, \quad \forall t \geq t_{0}+T_{0},
$$

where $z\left(t, t_{0}, z_{0}\right)$ is the solution of (11) with initial condition $z\left(t_{0}\right)=z_{0}$.

Choose constant $\alpha_{0}$ as follows:

$$
0<\alpha_{0} \leq \min \left\{\varepsilon_{0}, \frac{\delta_{0}}{a_{21}^{m}\left(F^{m}+S^{m}\right)+1}\right\} .
$$

Consider the following nonautonomous linear equation:

$$
\frac{d u(t)}{d t}=\mu(t) F(t)-d_{2}(t) u(t)
$$

From Corollary 5, for $\alpha_{0}$ and $M$ given in above there exist constants $\delta_{1}=\delta_{1}\left(\alpha_{0}\right)<\alpha_{0}$ and $T_{1}=T_{1}\left(\alpha_{0}, M\right)>0$ such that for any $t_{0} \in R_{+}$and $u_{0} \in[0, M]$, when $|\mu(t) F(t)|<\delta_{1}$ for all $t \geq t_{0}$, we have

$$
\left|u\left(t, t_{0}, u_{0}\right)\right|<\alpha_{0}, \quad \forall t \geq t_{0}+T_{1} .
$$

Let $\alpha_{1}=\min \left\{\varepsilon_{0}, \delta_{1} /\left(\mu^{m}+1\right)\right\}$, we will discuss the following three cases.

Case 1 . There exists a constant $T^{\prime} \geq T_{0}$ such that $F(t) \leq \alpha_{1}$ for all $t \geq T^{\prime}$.

Case 2. There exists a constant $T^{\prime} \geq T_{0}$ such that $F(t) \geq \alpha_{1}$ for all $t \geq T^{\prime}$.

Case 3. There exists a time sequence $\left\{\left[s_{k}, t_{k}\right]\right\}$ satisfying $T_{0} \leq$ $s_{1}<t_{1}<s_{2}<t_{2}<\cdots<s_{k}<t_{k}<\cdots$, and $\lim _{k \rightarrow \infty} s_{k}=\infty$ such that

$$
\begin{aligned}
& F(t) \leq \alpha_{1}, \quad \forall t \in \bigcup_{k=1}^{\infty}\left[s_{k}, t_{k}\right], \\
& F(t)>\alpha_{1}, \quad \forall t \notin \bigcup_{k=1}^{\infty}\left[s_{k}, t_{k}\right] .
\end{aligned}
$$

If Case 1 appears, we have

$$
\frac{d S(t)}{d t} \leq \mu(t) F(t)-d_{2}(t) S(t)
$$

for all $t \geq T^{\prime}$. Considering the auxiliary system

$$
\frac{d u(t)}{d t}=\mu(t) F(t)-d_{2}(t) u(t)
$$

Let $S(t)$ be the solution of the above equation satisfying initial condition $S\left(T^{\prime}\right)=u\left(T^{\prime}\right)$, by the comparison theorem, we have $S(t) \leq u(t)$ for all $t \geq T^{\prime}$. Since $F(t) \leq \alpha_{1}$ for all $t \geq T^{\prime}$, Hence, $|\mu(t) F(t)|<\delta_{1}$ for all $t \geq T^{\prime}$ and $u\left(T^{\prime}\right) \leq M$. By (41), we have $u(t)=u\left(t, T^{\prime}, u_{0}\left(T^{\prime}\right)\right)<\alpha_{0}$ for all $t \geq T^{\prime}+T_{1}$. Then, we obtain $S(t)<\alpha_{0}$ for all $t \geq T^{\prime}+T_{1}$. So,

$$
F(t) \leq \alpha_{1}, \quad S(t)<\alpha_{0}, \quad Z(t)<M, \quad \forall t \geq T^{\prime}+T_{1} .
$$

Hence, $a_{21}(t)(F(t)+S(t))<\delta_{0}$ for all $t \geq T^{\prime}+T_{1}$. In (38), choosing $t_{0}=T^{\prime}+T_{1}, Z_{0}=Z\left(T^{\prime}+T_{1}\right)$ and $Z(t)=$ $Z\left(t, t_{0}, Z\left(T^{\prime}+T_{1}\right)\right)$, by (38), we can get

$$
Z\left(t, t_{0}, Z\left(T^{\prime}+T_{1}\right)\right)<Z_{0}(t)+\varepsilon_{0}, \quad \forall t \geq T^{\prime}+T_{1}+T_{0} .
$$

Then,

$$
\begin{array}{r}
F(t) \leq \alpha_{1}<\varepsilon_{0}, \quad S(t)<\alpha_{0}<\varepsilon_{0}, \quad Z(t)<Z_{0}(t)+\varepsilon_{0}, \\
\forall t \geq T^{\prime}+T_{1}+T_{0} .
\end{array}
$$

For any $t \geq T^{\prime}+T_{1}+T_{0}$, we have

$$
\begin{aligned}
& \frac{d F(t)}{d t} \\
& =F(t)\left(b_{1}(t)-a_{11}(t)(F(t)+S(t))-\mu(t)-a_{12}(t) Z(t)\right) \\
& \geq F(t)\left(b_{1}(t)-2 \varepsilon_{0} a_{11}(t)-\mu(t)-a_{12}(t)\left(Z_{0}(t)+\varepsilon_{0}\right)\right) .
\end{aligned}
$$


Integrating the above inequality from $T^{\prime}+T_{1}+T_{0}$ to $t>T^{\prime}+$ $T_{1}+T_{0}$, we can obtain

$$
\begin{aligned}
F(t) \geq & F\left(T^{\prime}+T_{1}+T_{0}\right) \\
& \times \exp \int_{T^{\prime}+T_{1}+T_{0}}^{t}\left(b_{1}(\theta)-2 \varepsilon_{0} a_{11}(\theta)\right. \\
& \left.-\mu(\theta)-a_{12}(\theta)\left(Z_{0}(\theta)+\varepsilon_{0}\right)\right) d \theta .
\end{aligned}
$$

From this and (35), it follows $\lim _{t \rightarrow \infty} F(t)=\infty$ which leads to a contradiction.

If Case 2 appears, then obviously $F(t)$ is permanent.

If Case 3 appears, for any $\left\{\left[s_{k}, t_{k}\right]\right\}$ we have $F\left(s_{k}\right)=$ $F\left(t_{k}\right)=\alpha_{1}$ and $F(t) \leq \alpha_{1}$ for all $t \in\left[s_{k}, t_{k}\right]$. If $t_{k}-s_{k} \leq T_{1}+T_{0}$, choosing constant

$$
h=\sup _{t \geq 0}\left\{b_{1}(t)+a_{11}(t)\left(\varepsilon_{0}+M\right)+\mu(t)+a_{12}(t) M\right\},
$$

integrating the first equation of model (1) in interval $\left[s_{k}, t_{k}\right]$, we get

$$
\begin{aligned}
& \frac{d F(t)}{d t}=F\left(s_{k}\right) \exp \int_{s_{k}}^{t}\left(b_{1}(\theta)-a_{11}(\theta)(F(\theta)+S(\theta))\right.\left.-\mu(\theta)-a_{12}(\theta) Z(\theta)\right) d \theta \\
& \geq F\left(s_{k}\right) \exp \int_{s_{k}}^{t}\left(b_{1}(\theta)-a_{11}(\theta)\left(\varepsilon_{0}+M\right)\right. \\
&\left.\quad-\mu(\theta)-a_{12}(\theta) M\right) d \theta \\
& \geq \alpha_{1} \exp \left\{-h\left(T_{1}+T_{0}\right)\right\} .
\end{aligned}
$$

If $t_{k}-s_{k}>T_{1}+T_{0}$, because $F(t) \leq \alpha_{1}$ for all $t \in\left[s_{k}, t_{k}\right]$, we have $|\mu(t) F(t)|<\delta_{1}$ for all $t \in\left[s_{k}, t_{k}\right]$ and $u\left(s_{k}\right)=S\left(s_{k}\right) \leq M$. Hence, we have $u(t)<\alpha_{0}$ for all $t \in\left[s_{k}+T_{1}, t_{k}\right]$. Then, we obtain $S(t)<\alpha_{0}$ for all $t \in\left[s_{k}+T_{1}, t_{k}\right]$. So,

$$
\begin{array}{r}
F(t) \leq \alpha_{1}, \quad S(t)<\alpha_{0}, \quad Z(t)<M \\
\forall t \in\left[s_{k}+T_{1}, t_{k}\right] .
\end{array}
$$

Hence, $a_{21}(t)(F(t)+S(t))<\delta_{0}$ for any $t \in\left[s_{k}+T_{1}, t_{k}\right]$. In (38), choosing $t_{0}=s_{k}+T_{1}, Z_{0}=Z\left(s_{k}+T_{1}\right)$ and $Z(t)=$ $Z\left(t, t_{0}, Z\left(s_{k}+T_{1}\right)\right)$, by (38), we can get

$$
Z\left(t, t_{0}, Z\left(s_{k}+T_{1}\right)\right)<Z_{0}(t)+\varepsilon_{0}, \quad \forall t \in\left[s_{k}+T_{1}+T_{0}, t_{k}\right] .
$$

Then,

$$
\begin{aligned}
& F(t) \leq \alpha_{1}<\varepsilon_{0}, \quad S(t)<\alpha_{0}<\varepsilon_{0}, \quad Z(t)<Z_{0}(t)+\varepsilon_{0}, \\
& \forall t \in\left[s_{k}+T_{1}+T_{0}, t_{k}\right] \text {. }
\end{aligned}
$$

For any $t \in\left[s_{k}, t_{k}\right]$, when $t \leq s_{k}+T_{1}+T_{0}$, we can obtain from the above discussion on the case $t_{k}-s_{k} \leq T_{1}+T_{0}$,

$$
F(t) \geq \alpha_{1} \exp \left\{-h\left(T_{1}+T_{0}\right)\right\} \text {. }
$$

In particular, we have $F\left(s_{k}+T_{1}+T_{0}\right) \geq \alpha_{1} \exp \left\{-h\left(T_{1}+T_{0}\right)\right\}$. When $t>s_{k}+T_{1}+T_{0}$, then we choose an integer $p>0$ such that $t \in\left[s_{k}+T_{1}+T_{0}+p \lambda, s_{k}+T_{1}+T_{0}+(p+1) \lambda\right]$; integrating the first equation of system (1) from $s_{k}+T_{1}+T_{0}$ to $t>s_{k}+T_{1}+T_{0}$ we can obtain

$$
\begin{aligned}
& \frac{d F(t)}{d t} \\
& =F\left(s_{k}+T_{1}+T_{0}\right) \\
& \quad \times \exp \int_{s_{k}+T_{1}+T_{0}}^{t}\left(b_{1}(\theta)-a_{11}(\theta)(F(\theta)+S(\theta))\right. \\
& \left.\quad-\mu(\theta)-a_{12}(\theta) Z(\theta)\right) d \theta \\
& \geq \alpha_{1} \exp \left\{-h\left(T_{1}+T_{0}\right)\right\} \\
& \quad \times \exp \int_{s_{k}+T_{1}+T_{0}}^{t}\left(b_{1}(\theta)-2 \varepsilon_{0} a_{11}(\theta)-\mu(\theta)\right. \\
& =\alpha_{1} \exp \left\{-h\left(T_{1}+T_{0}\right)\right\} \\
& \quad \times \exp \left(\int_{s_{k}+T_{1}+T_{0}}^{s_{k}+T_{1}+T_{0}+p \lambda}+\int_{s_{k}+T_{1}+T_{0}+p \lambda}^{t}\left(Z_{0}(\theta)+\varepsilon_{0}\right)\right) d \theta \\
& \quad \times\left(b_{1}(\theta)-2 \varepsilon_{0} a_{11}(\theta)\right. \\
& \left.\quad-\mu(\theta)-a_{12}(\theta)\left(Z_{0}(\theta)+\varepsilon_{0}\right)\right) d \theta \\
& \geq
\end{aligned}
$$

where $h_{1}=\sup _{t \geq 0}\left\{b_{1}(t)+2 \varepsilon_{0} a_{11}(t)+\mu(t)+a_{12}(t)\left(Z_{0}(\theta)+\varepsilon_{0}\right)\right\}$. Choose

$$
m_{1}=\alpha_{1} \exp \left\{-\left(h\left(T_{1}+T_{0}\right)+h_{1} \lambda\right)\right\} ;
$$

then from above discussion we finally obtain

$$
F(t) \geq m_{1}, \quad \forall t \in \bigcup_{k=1}^{\infty}\left[s_{k}, t_{k}\right] .
$$

In addition, we have $F(t)>\alpha_{1}$ for all $t \notin \bigcup_{k=1}^{\infty}\left[s_{k}, t_{k}\right]$. Then, we finally obtain

$$
F(t) \geq m_{1}, \quad \forall t \geq T^{\prime} .
$$

Considering the second equation of system (1), according to Theorem 6, we have

$$
\begin{aligned}
\frac{d S(t)}{d t}= & \mu(t) F(t)-d_{2}(t) S(t)-a_{11}(t) \\
& \times(F(t)+S(t)) S(t)-a_{12}(t) S(t) Z(t) \\
\geq & \mu(t) m_{1}-\left(d_{2}(t)+2 M a_{11}(t)+a_{12}(t) M\right) S(t)
\end{aligned}
$$

for all $t \geq T^{\prime}$.

Considering the auxiliary equation

$$
\frac{d u(t)}{d t}=\mu(t) m_{1}-\left(d_{2}(t)+2 M a_{11}(t)+a_{12}(t) M\right) u(t) .
$$


According to Lemma 3, there exists a constant $m_{2}$ such that $\lim \inf _{t \rightarrow \infty} u(t) \geq m_{2}$ for any positive solution of (61). By the comparison theorem and (60), we have

$$
\liminf _{t \rightarrow \infty} S(t) \geq \liminf _{t \rightarrow \infty} u(t) \geq m_{2}
$$

Let $m=\min \left\{m_{1}, m_{2}\right\}$; from (59) and (62) we obtain

$$
\liminf _{t \rightarrow \infty} F(t) \geq m, \quad \liminf _{t \rightarrow \infty} S(t) \geq m
$$

This completes the proof.

Theorem 9. Suppose that $\left(H_{1}\right)$ and $\left(H_{2}\right)$ hold and there exists a constant $\lambda>0$ such that

$$
\limsup _{t \rightarrow \infty} \int_{t}^{t+\lambda}\left(b_{1}(\theta)-\mu(\theta)-a_{12}(\theta) Z_{0}(\theta)\right) d \theta \leq 0 .
$$

Then,

$$
\lim _{t \rightarrow \infty} F(t)=0, \quad \lim _{t \rightarrow \infty} S(t)=0
$$

for any positive solution $(F(t), S(t), Z(t))$ of system (1).

The biological meaning of Theorem 9 is that if $\left(\mathrm{H}_{1}\right),\left(\mathrm{H}_{2}\right)$, and (64) hold, the prey species will be extinct. Form the viewpoint of the Nature Conservancy and Human Health, the best way for our human beings is to keep the existence of the species, and, meanwhile, guarantee such existence do no harm to us. Thus, the condition of making harmful rat extinct for management expert is very important. Therefore, it is a critical threshold value.

Proof. By (64), we have for any $0<\varepsilon<1$, there are positive constants $\varepsilon_{1}<\varepsilon$ and $\varepsilon_{0}$ and $T_{0}>0$ such that

$$
\int_{t}^{t+\lambda}\left(b_{1}(\theta)-\mu(\theta)-a_{11}(\theta) \varepsilon-a_{12}(\theta)\left(Z_{0}(\theta)-\varepsilon_{1}\right)\right) d \theta<-\varepsilon_{0}
$$

for all $t \geq T_{0}$. From the third equation of system (1) we have

$$
\frac{d Z(t)}{d t} \geq Z(t)\left(b_{2}(t)-a_{22}(t) Z(t)\right), \quad \forall t \geq T_{0} ;
$$

applying the comparison theorem and conclusion (b) of Lemma 1 , there exists a constant $T_{1} \geq T_{0}$ such that $Z(t) \geq$ $Z_{0}(t)-\varepsilon_{1}$ for all $t \geq T_{1}$. For any $t \geq T_{1}$, we have

$$
\begin{aligned}
& \frac{d F(t)}{d t} \\
& =F(t)\left(b_{1}(t)-a_{11}(t)(F(t)+S(t))-\mu(t)-a_{12}(t) Z(t)\right) \\
& \leq F(t)\left(b_{1}(t)-a_{11}(t) F(t)-\mu(t)-a_{12}(t) Z(t)\right) .
\end{aligned}
$$

For any $0<\varepsilon<1$, if $F(t) \geq \varepsilon$ for all $t \geq T_{1}$, integrating (68) from $T_{1}$ to $t$, we obtain

$$
\begin{aligned}
F(t) \leq F\left(T_{1}\right) \exp \int_{T_{1}}^{t}( & b_{1}(\theta)-a_{11}(\theta) \varepsilon-\mu(\theta) \\
& \left.-a_{12}(\theta)\left(Z_{0}(\theta)-\varepsilon_{1}\right)\right) d \theta .
\end{aligned}
$$

From (66), it follows that $F(t) \rightarrow 0$ as $t \rightarrow \infty$ which leads to a contradiction. Hence, there exists a $t_{1} \geq T_{1}$ such that $F\left(t_{1}\right)<\varepsilon$. Let

$$
h=\sup _{t \geq T_{1}}\left\{b_{1}(t)+a_{11}(t)+\mu(t)+a_{12}(t)\left(Z_{0}(\theta)-\varepsilon_{1}\right)\right\} ;
$$

we prove

$$
F(t) \leq \varepsilon \exp \{h \lambda\}, \quad \forall t \geq t_{1} .
$$

If (71) is not true, then there exists a $t_{2}>t_{1}$ such that $F\left(t_{2}\right)>$ $\varepsilon \exp \{h \lambda\}$. From $F\left(t_{1}\right)<\varepsilon$, there exists a $t_{3} \in\left(t_{1}, t_{2}\right)$ such that $F\left(t_{3}\right)=\varepsilon$ and $F(t)>\varepsilon$ for all $t \in\left(t_{3}, t_{2}\right)$. Let $p \geq 0$ be an integer such that $t_{2} \in\left(t_{3}+p \lambda, t_{3}+(p+1) \lambda\right]$, integrating (68) from $t_{3}$ to $t_{2}$,

$$
\begin{aligned}
\varepsilon \exp \{h \lambda\}< & F\left(t_{2}\right) \leq F\left(t_{3}\right) \\
& \quad \times \exp \int_{t_{3}}^{t_{2}}\left(b_{1}(\theta)-a_{11}(\theta) \varepsilon\right. \\
& \left.\quad-\mu(\theta)-a_{12}(\theta)\left(Z_{0}(\theta)-\varepsilon_{1}\right)\right) d \theta \\
\leq & \varepsilon \exp \{h \lambda\},
\end{aligned}
$$

which leads to a contradiction. Hence, (71) holds. From the arbitrariness of $\varepsilon$, we finally obtain $F(t) \rightarrow 0$ as $t \rightarrow \infty$. Considering the second equation

$$
\begin{aligned}
\frac{d S(t)}{d t} \\
=\mu(t) F(t)-d_{2}(t) S(t)-a_{11}(t) \\
\quad \times(F(t)+S(t)) S(t)-a_{12}(t) S(t) Z(t) \\
\leq \mu(t) F(t)-d_{2}(t) S(t) \leq \varepsilon \exp \{h \lambda\} \mu(t)-d_{2}(t) S(t)
\end{aligned}
$$

for all $t \geq t_{1}$. Using Corollary 5 , we can easily obtain $S(t) \rightarrow 0$ as $t \rightarrow \infty$. This completes the proof.

Further, from conclusion (c) of Lemma 1, as consequence of Theorems 8 and 9 , we also have the following corollaries.

Corollary 10. Suppose that $\left(H_{1}\right)$ and $\left(H_{2}\right)$ hold, $a_{22}^{l}>0$ and there exists a constant $\lambda>0$ such that

$$
\liminf _{t \rightarrow \infty} \int_{t}^{t+\lambda}\left(b_{1}(\theta)-\mu(\theta)-a_{12}(\theta)\left(\frac{b_{2}}{a_{22}}\right)^{m}\right) d \theta>0 .
$$

Then,

$$
\liminf _{t \rightarrow \infty} F(t)>m, \quad \liminf _{t \rightarrow \infty} S(t)>m
$$

for any positive solution $(F(t), S(t), Z(t))$ of system (1). 




(a)

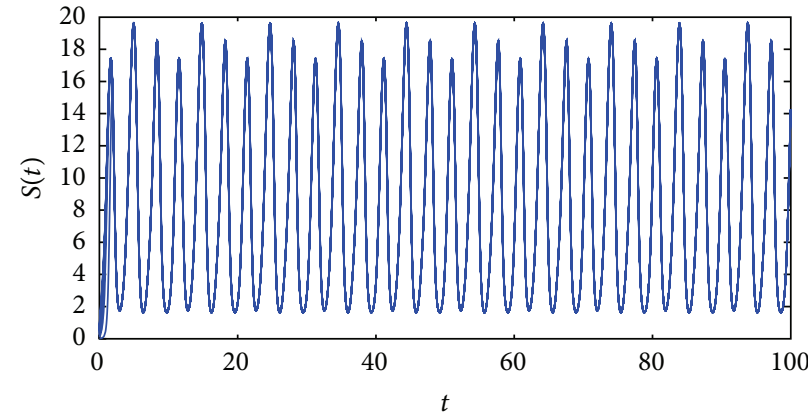

(b)

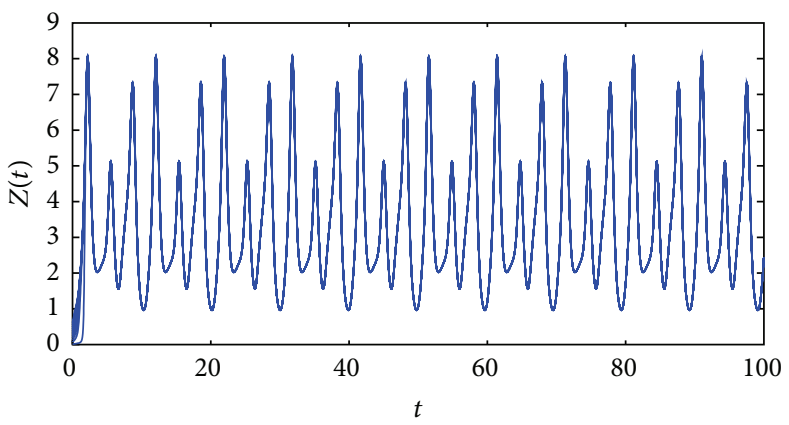

(c)

Figure 1: Permanence of system (1) with parameters in Example 1.

Corollary 11. Suppose that $\left(H_{1}\right)$ and $\left(H_{2}\right)$ hold, $a_{22}^{l}>0$ and there exists a constant $\lambda>0$ such that

$$
\limsup _{t \rightarrow \infty} \int_{t}^{t+\lambda}\left(b_{1}(\theta)-\mu(\theta)-a_{12}(\theta)\left(\frac{b_{2}}{a_{22}}\right)^{l}\right) d \theta \leq 0 .
$$

Then,

$$
\lim _{t \rightarrow \infty} F(t)=0, \quad \lim _{t \rightarrow \infty} S(t)=0
$$

for any positive solution $(F(t), S(t), Z(t))$ of system (1).

As consequences of Theorems 8 and 9, we have the following corollaries. Firstly, from Lemmas 1 we obtain that if $\left(\mathrm{H}_{1}\right)$ and $\left(\overline{\mathrm{H}_{2}}\right)$ hold, then (31) have the globally uniformly attractive nonnegative $\omega$-periodic solutions $Z_{0}(t)$.

Corollary 12. Suppose that system (1) is $\omega$-periodic and $\left(H_{1}\right)$ and $\left(\overline{H_{2}}\right)$ hold. Then the fertility prey $F(t)$ and infertility prey $S(t)$ in model (1) are permanent if and only if

$$
\bar{\mu}<\overline{b_{1}-a_{12} Z_{0}} .
$$

Corollary 13. Suppose that system (1) is $\omega$-periodic and $\left(H_{1}\right)$ and $\left(\overline{H_{2}}\right)$ hold. Then the fertility prey $F(t)$ and infertility prey $S(t)$ in model (1) are extinct if and only if

$$
\bar{\mu} \geq \overline{b_{1}-a_{12} Z_{0}} .
$$

Remark 14. In the process of the prevention and control of rat in the grasslands and farmland, we are concerned about how many biological sterile drug should be put in a period in order to make the population of the harmful rat reduce to a very low level. From Corollaries 12 and 13, we can easily obtain that $\bar{\mu}=\overline{b_{1}-a_{12} Z_{0}}$ is a critical value. If $\bar{\mu} \geq \overline{b_{1}-a_{12} Z_{0}}$, we can control the population of rat at a very low level. The results are very meaningful and significant.

When system (1) is simplified into the corresponding autonomous system, that is,

$$
\begin{aligned}
\frac{d F(t)}{d t}= & F(t)\left(b_{1}-a_{11}(F(t)+S(t))-\mu-a_{12} Z(t)\right), \\
\frac{d S(t)}{d t}= & \mu F(t)-d_{2} S(t)-a_{11}(F(t)+S(t)) S(t) \\
& -a_{12} S(t) Z(t), \\
\frac{d Z(t)}{d t}= & Z(t)\left(b_{2}+a_{21}(F(t)+S(t))-a_{22} Z(t)\right) .
\end{aligned}
$$

Remark 15. For system (80), we know that $\mu=b_{1}-a_{12} b_{2} / a_{22}$ is a critical value. Then, we can obtain that the quantity of biological sterile drug should be $\mu \geq b_{1}-a_{12} b_{2} / a_{22}$.

\section{Example and Numerical Simulation}

In this section, we give some examples and numerical simulations to the above theoretical analysis. 


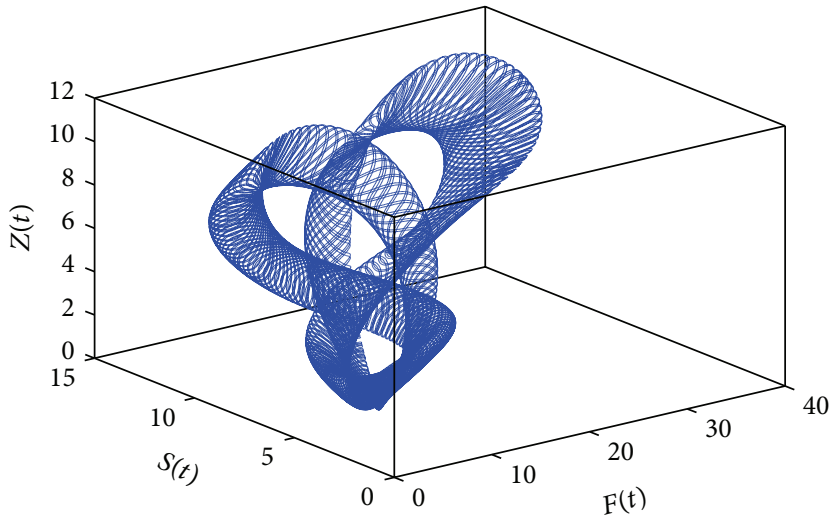

(a)

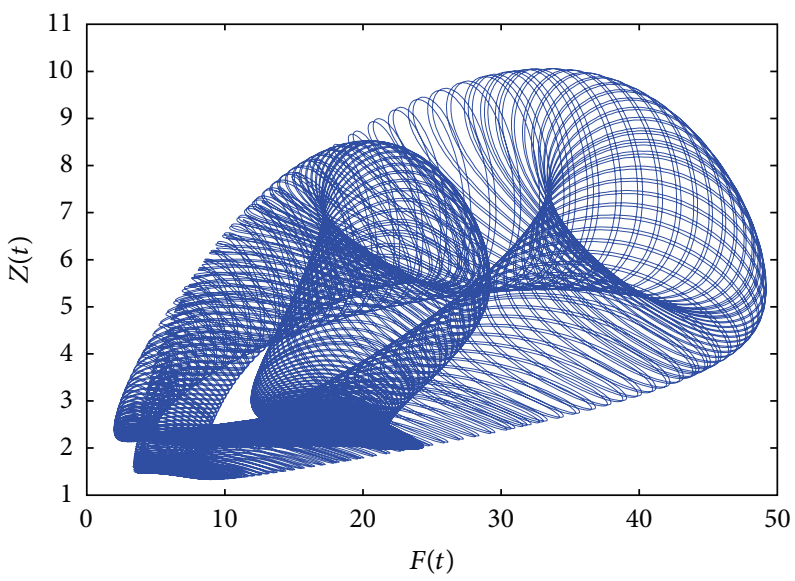

(c)

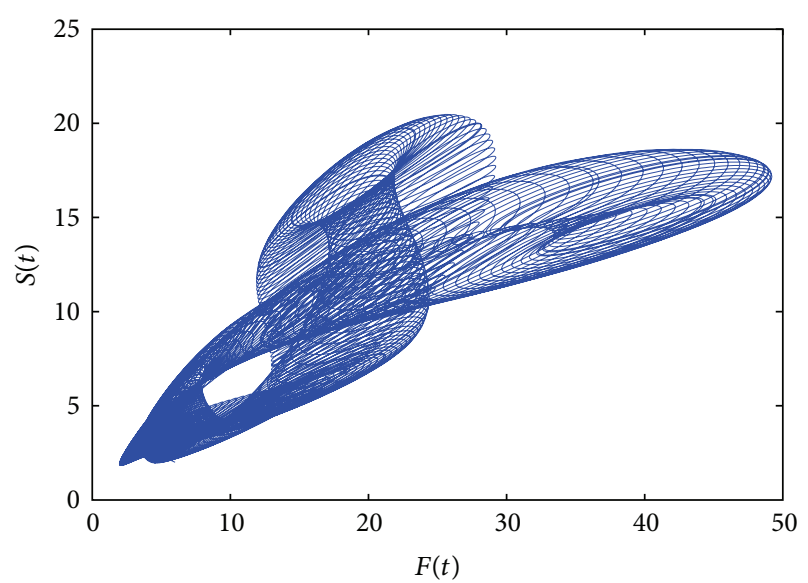

(b)

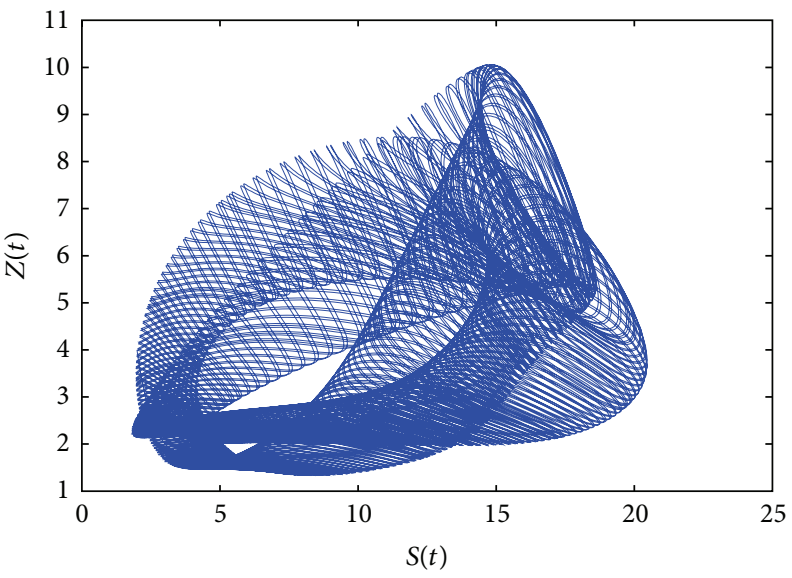

(d)

FIGURE 2: Chaotic behavior of system (1) with parameters in Example 2.

Example 1. Take $b_{1}(t)=10+\sin (6 t / \pi), a_{11}(t)=0.09+0.001 \times$ $\sin (6 t / \pi), \mu(t)=9 \times(0.35+0.2 \times \cos (4 t / \pi)+0.01 \times \sin (4 t / \pi))$, $a_{12}(t)=1.2+\cos (6 t / \pi), d_{2}(t)=2+0.005 \times \sin (t \pi / 6), b_{2}(t)=$ $1.5+\cos (6 t / \pi), a_{21}(t)=0.7+0.3 \times \cos (6 t / \pi)$ and $a_{22}(t)=5+$ $3 \times \sin (6 t / \pi)$ in system (1). We easily verify that assumptions $\left(\mathrm{H}_{1}\right),\left(\mathrm{H}_{2}\right)$ hold. From Lemma 1, some fixed positive solution $Z_{0}(t)$ of system (32) satisfies $0.00625 \leq Z_{0}(t) \leq 1.25$. Moreover, condition (35) $\liminf _{t \rightarrow \infty} \int_{t}^{t+\lambda}\left(b_{1}(\theta)-\mu(\theta)-\right.$ $\left.a_{12}(\theta) Z_{0}((\theta))\right) d \theta>0>9-9 \times(0.35+0.21)-2.2 \times 1.25=$ $1.21>0$ holds, therefore, by Theorem 8 , system (1) with these parameters is permanent. The corresponding numerical simulations are given in Figure 1, and this figure illustrates that the solutions will tend towards periodic oscillation along with time passing. It means that there exists a periodic solution, and it is seemed that this periodic solution is globally attractive.

Example 2. Take $b_{1}(t)=10+\sin (6 t / \pi), a_{11}(t)=0.09+0.001 \times$ $\sin (6 t / \pi), \mu(t)=9 \times(0.35+0.1 \times \cos (t / 4)+0.1 \times \sin (t / 4))$, $a_{12}(t)=1.2+\cos (t / 6), d_{2}(t)=2+0.005 \times \sin (6 t / \pi)$, $b_{2}(t)=1.5+\cos (t / 6), a_{21}(t)=0.7+0.3 \times \cos (6 t / \pi)$ and $a_{22}(t)=5+3 \times \sin (t / 6)$ in system (1). By similar calculation, we can obtain that $\left(\mathrm{H}_{1}\right),\left(\mathrm{H}_{2}\right)$ and (35) hold. Therefore, by
Theorem 8 , system (1) with these parameters is permanent, as shown in numerical simulations of Figures 2(a)-2(d), which not only illustrate the validity of the proposed results, but also display the interesting complex dynamic behaviors; that is, there is not periodic oscillation along with time passing as like Figure 1, and from (a)-(d) in Figure 2, it can be obviously seen that there is a strange chaotic attractor, which may contribute to a better understanding of the complex chaotic behaviors which can be a high risk of the uncertain number of the population due to the unpredictability.

\section{Conclusion}

Based on the mouse rampant phenomenon in some areas, a predator-prey model with infertility control in rat species is established in the situation where all coefficients depend on time. For the nonautonomous system threshold conditions for the permanence and the extinction of fertility prey and infertility prey are established. The condition for permanence has the form of a lim inf condition for some time-dependent sterility conversion rate $(\mu(t))$ while the condition for extinction assumes the form of a lim sup condition. Hence, in 
the general case the main results are not threshold criteria in a strict sense. However, in the periodic cases, the conditions merge into a sharp threshold criterion and sterile drug dosage can be obtained. Two numerical examples are carried out to support theoretical results, and the second simulation result suggests that there may be interesting dynamic behaviors in this model-a strange chaotic attractor. Furthermore, chaos may cause the number of pests approaching to the uncontrollable state due to the unpredictability. Thus, how to control chaos in the population model is very important, which needs further investigation.

\section{Conflict of Interests}

The authors declare that they have no financial and personal relationships with other people or organizations that can inappropriately influence their work, there is no professional or other personal interests of any nature or kind in any product, service, and/or company that could be construed as influencing the position presented in, or the review of, this paper.

\section{Acknowledgments}

This work was supported by The National Natural Science Foundation of China [11271312,11371313,11241005], The Natural Science Foundation of Shanxi Province [20130110025], and the Research Project at Yuncheng University [XK2012001,XK2012007].

\section{References}

[1] Hainan Online News Center, "Inner Mongolia steppe: starving rat injury by oneself and alse eat live sheep," 2005, http://news.hainan.net/newshtml/2005w6r9/23094f1.htm.

[2] Modern Express, "Inner Mongolia steppe introduced eleven Yinhu in order to destroy rat," 2006, http://kb.dsqq.cn/old/ html/2006-11/23/content_49946823.htm.

[3] "Inner Mongolia DongWuZhMuQinQi farming and animal husbandry information network," 2011, http://www.nmgdwzmqq.agri.gov.cn/sites/MainSite/.

[4] C. M. Hardy, L. A. Hinds, P. J. Kerr et al., "Biological control of vertebrate pests using virally vectored immunocontraception," Journal of Reproductive Immunology, vol. 71, no. 2, pp. 102-111, 2006.

[5] A. D. Arthur, R. P. Pech, and G. R. Singleton, "Cross-strain protection reduces effectiveness of virally vectored fertility control: results from individual-based multistrain models," Journal of Applied Ecology, vol. 44, no. 6, pp. 1252-1262, 2007.

[6] A. T. Rutberg, R. E. Naugle, L. A. Thiele, and I. K. M. Liu, "Effects of immunocontraception on a suburban population of whitetailed deer Odocoileus virginianus," Biological Conservation, vol. 116, no. 2, pp. 243-250, 2004.

[7] C. K. Williams, C. C. Davey, R. J. Moore et al., "Population responses to sterility imposed on female European rabbits," Journal of Applied Ecology, vol. 44, no. 2, pp. 291-301, 2007.

[8] J. Jacob, Rahmini, and Sudarmaji, "The impact of imposed female sterility on field populations of ricefield rats (Rattus argentiventer)," Agriculture, Ecosystems \& Environment, vol. 115, no. 1-4, pp. 281-284, 2006.
[9] Z. Zhang, "Mathematical models of wildlife management by contraception," Ecological Modelling, vol. 132, no. 1-2, pp. 105$113,2000$.

[10] Z. Teng and Z. Li, "Permanence and asymptotic behavior of the $N$-species nonautonomous lotka-volterra competitive systems," Computers \& Mathematics with Applications, vol. 39, no. 7-8, pp. 107-116, 2000.

[11] X. Niu, T. Zhang, and Z. Teng, "The asymptotic behavior of a nonautonomous eco-epidemic model with disease in the prey," Applied Mathematical Modelling, vol. 35, no. 1, pp. 457-470, 2011.

[12] T. Zhang and Z. Teng, "On a nonautonomous SEIRS model in epidemiology," Bulletin of Mathematical Biology, vol. 69, no. 8, pp. 2537-2559, 2007.

[13] X. Feng, Z. Teng, and L. Zhang, "Permanence for nonautonomous n-species Lotka-Volterra competitive systems with feedback controls," Rocky Mountain Journal of Mathematics, vol. 38, no. 5, pp. 1355-1376, 2008. 


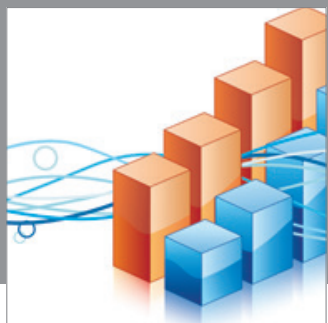

Advances in

Operations Research

mansans

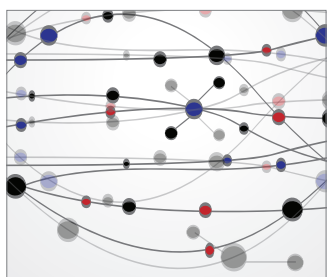

The Scientific World Journal
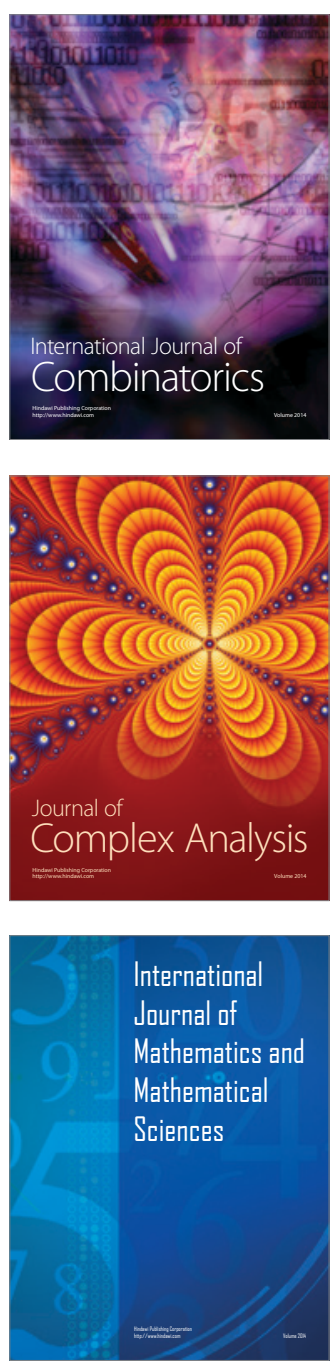
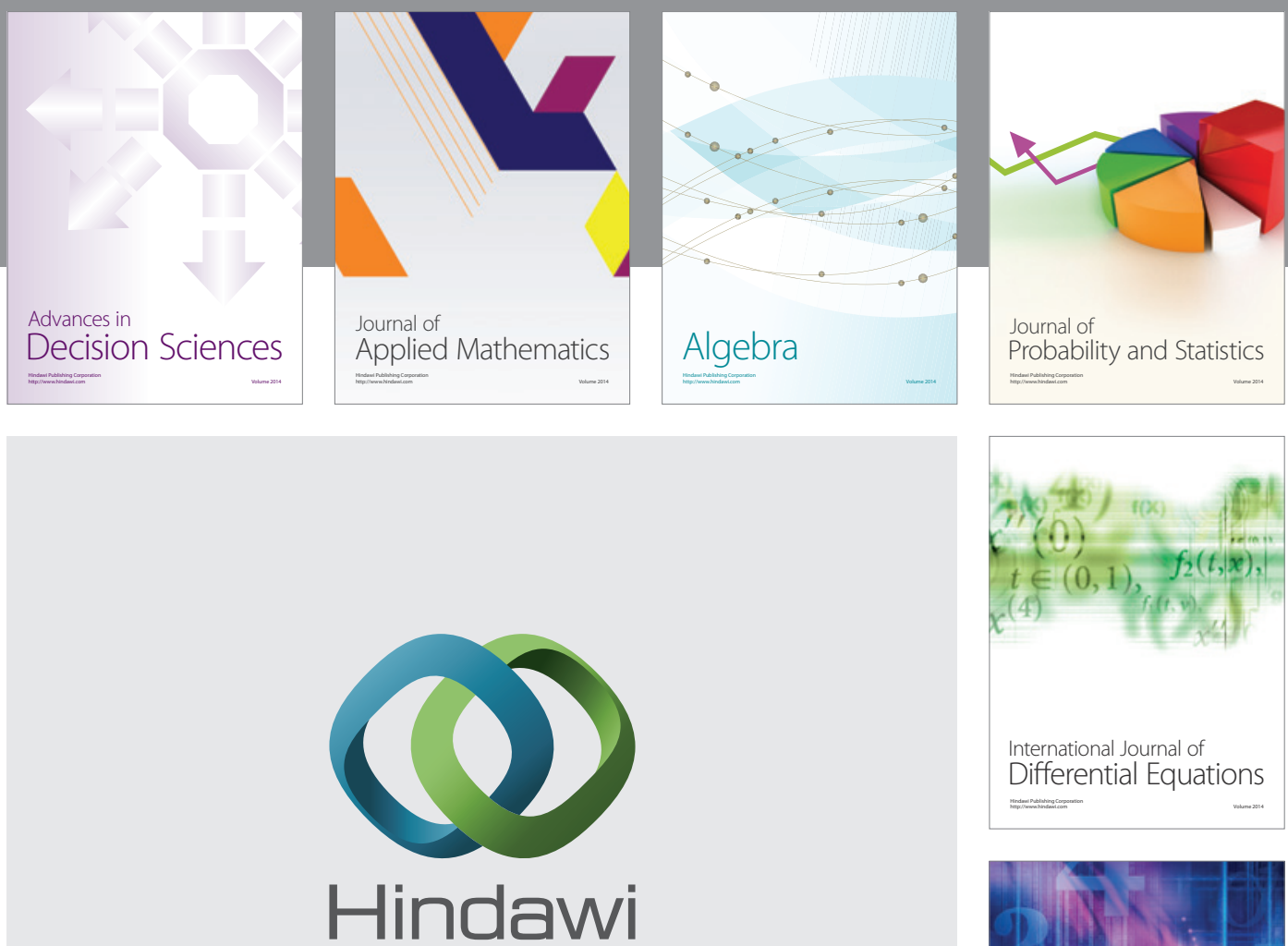

Submit your manuscripts at http://www.hindawi.com
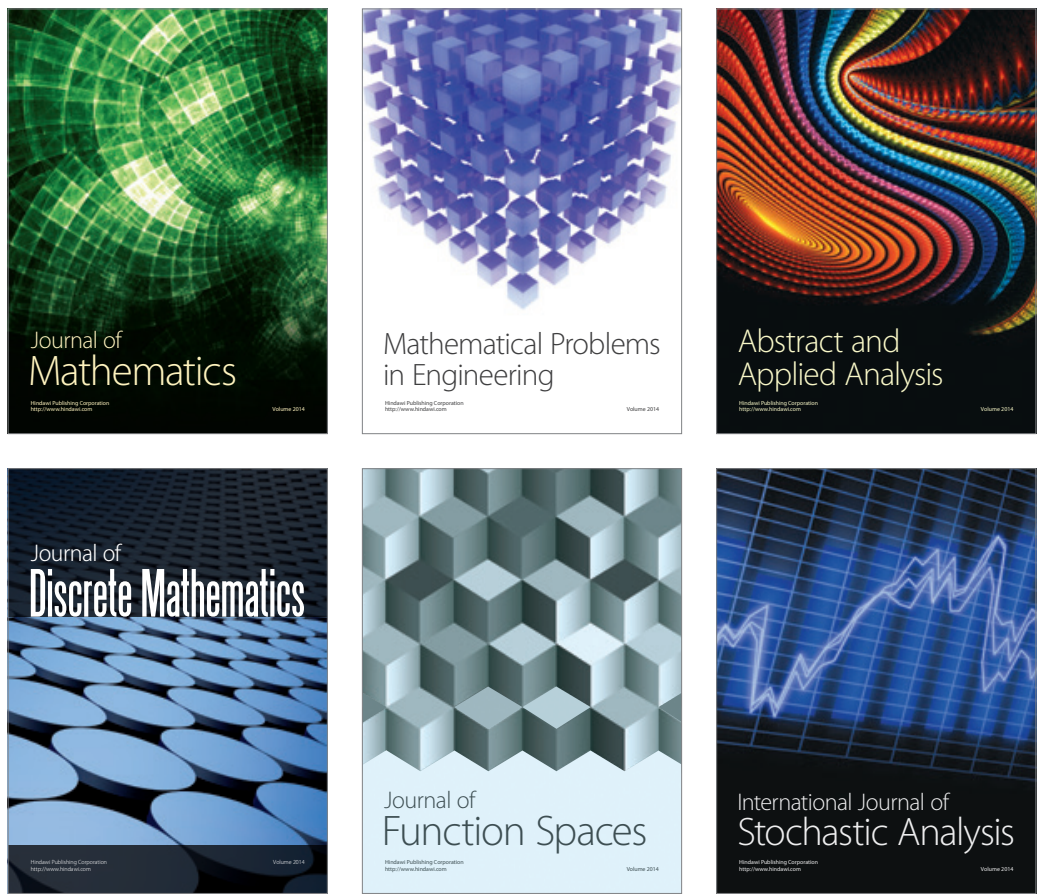

Journal of

Function Spaces

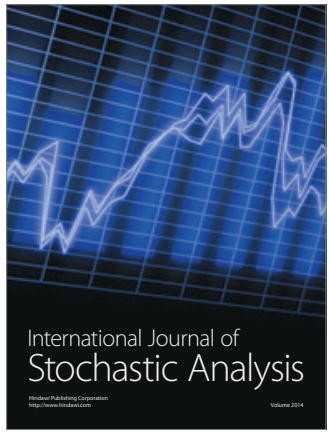

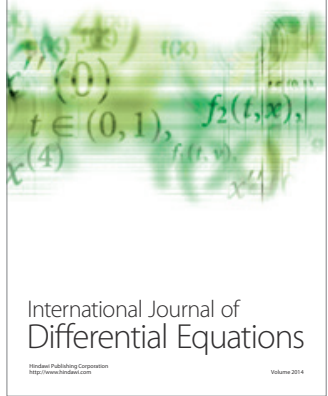
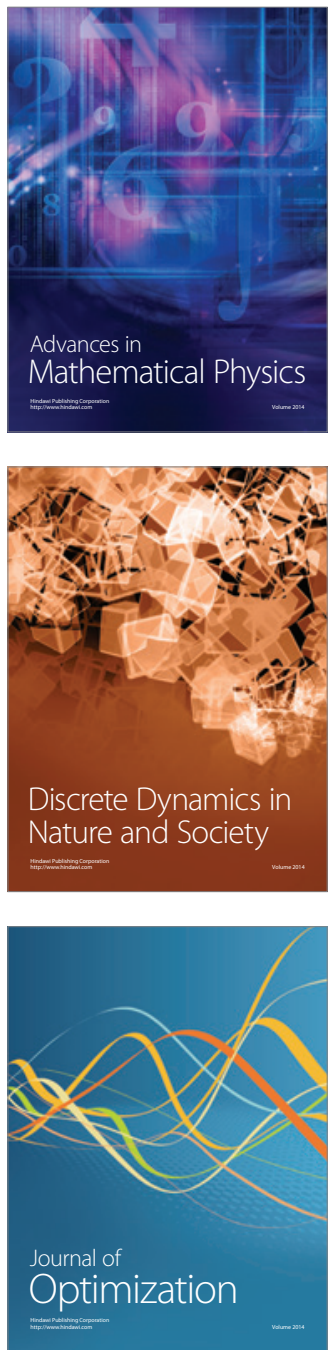